BMJ Open

Diabetes

Research

\& Care

\section{Breakfast skipping is associated with persistently increased arterial stiffness in patients with type 2 diabetes}

To cite: Mita T, Osonoi Y, Osonoi T, et al. Breakfast skipping is associated with persistently increased arterial stiffness in patients with type 2 diabetes. BMJ Open Diab Res Care 2020;8:e001162. doi:10.1136/ bmjdrc-2019-001162

- Additional material is published online only. To view, please visit the journal online (http://dx.doi.org/10.1136/ bmjdrc-2019-001162).

Received 27 December 2019 Revised 11 January 2020 Accepted 28 January 2020
Check for updates

(C) Author(s) (or their employer(s)) 2020. Re-use permitted under CC BY-NC. No commercial re-use. See rights and permissions. Published by BMJ.

For numbered affiliations see end of article.

Correspondence to Dr Tomoya Mita; tom-m@juntendo.ac.jp

\section{ABSTRACT}

Objective While certain lifestyle habits may be associated with arterial stiffness, there is limited literature investigating the relationship between lifestyle habits and longitudinal changes in arterial stiffness in patients with type 2 diabetes mellitus (T2DM). This is an exploratory study to determine whether lifestyle habits, in addition to conventional atherosclerotic risk factors, are associated with increased arterial stiffness.

Research design and methods The study participants comprised 734 Japanese outpatients with T2DM and no history of apparent cardiovascular diseases. Lifestyle habits were analyzed using self-reported questionnaires, and brachial-ankle pulse wave velocity (baPWV) was measured at baseline, and at years 2 and 5 . A multivariable linear mixedeffects model was used to determine the predictive value of lifestyle habits and possible atherosclerotic risk factors for longitudinal change in baPWV.

Results Over 5 years of follow-up, baPWV values significantly increased. In a multivariable linear mixedeffects model that adjusted for age and gender, a low frequency of breakfast intake was significantly associated with persistently high baPWV, independently of other lifestyle habits. Furthermore, in a multivariable linear mixed-effects model that included both lifestyle habits and possible atherosclerotic risk factors, a low frequency of breakfast intake remained the only independent predictive factor for persistently high baPWV. Subjects who ate breakfast less frequently tended to have additional unhealthy lifestyle habits and atherosclerotic risk factors. Conclusions Our analyses suggest that breakfast skipping is an independent lifestyle habit that is associated with persistently increased arterial stiffness in patients with T2DM. Trial registration number UMIN000010932.

\section{INTRODUCTION}

Patients with type two diabetes mellitus (T2DM) are at high risk of developing cardiovascular diseases (CVD), which are also one of the main causes of death in this population. ${ }^{1}$ In general, patients with T2DM have increased arterial stiffness, ${ }^{2}$ which may be one of the mechanisms linking T2DM to cardiovascular morbidity and mortality. ${ }^{3}$ In patients with T2DM, increased arterial stiffness is associated with the incidence of CVD. ${ }^{45}$ Identifying

\section{Significance of this study}

What is already known about this subject?

- Cross-sectional studies demonstrated that certain lifestyle habits such as poor sleep quality and low physical activity may be associated with arterial stiffness.

- A recent cross-sectional study demonstrated that breakfast skipping was associated with a higher prevalence of subclinical atherosclerosis in the general population, not in patents with type 2 diabetes mellitus (T2DM).

What are the new findings?

- A low frequency of breakfast intake was significantly associated with persistently high brachial-ankle pulse wave velocity independently of other lifestyle habits and possible atherosclerotic risk factors.

How might these results change the focus of research or clinical practice?

- Asking about the frequency of breakfast intake in clinical practice may be a simple and useful method to help identify patients at high risk of developing cardiovascular diseases.

- Eating breakfast more frequently may improve arterial stiffness in patients with T2DM.

patients with a high probability of developing CVD by evaluating arterial stiffness is important in order to reduce the morbidity and mortality of these diseases. The brachial-ankle pulse wave velocity (baPWV) is a non-invasive technique that is often used clinically to assess arterial wall stiffness, evaluate the severity of atherosclerosis and predict CVD in patients with T2DM. ${ }^{45}$

Previous cross-sectional studies demonstrated that increased arterial stiffness in patients with T2DM was associated with conventional atherosclerotic risk factors such as age, gender, body mass index (BMI), duration of T2DM, glycemic control, dyslipidemia, systolic blood pressure (BP), estimated glomerular filtration rate (eGFR), elevated uric acid levels and albuminuria. $^{267}$ Other recent cross-sectional 
studies showed that in addition to these conventional atherosclerotic risk factors, poor sleep quality and low physical activity was correlated with increased arterial stiffness in patients with T2DM. ${ }^{8-10}$ By assessing serial changes in arterial stiffness, a recent study demonstrated that chronic hyperglycemia, older age, female gender, increased systolic $\mathrm{BP}$ and pulse rate and the presence of dyslipidemia and retinopathy were associated with the progression of arterial stiffness in patients with T2DM. ${ }^{6}$ However, in that study, lifestyle-related problems were not assessed, and thus it remains largely unknown how factors such as energy intake, physical activity, sleep duration, sleep quality, chronotype, dietary habits such as skipping breakfast and/or eating latenight dinners and depressive state are associated with the progression of atherosclerosis.

Lifestyle habits have potential to cause arterial stiffness through deteriorating cardiometabolic risk profiles and/or altering hormone levels as follows. Previous reports demonstrated that increased counter-regulatory hormones due to duration of sleep and/or poor sleep quality may be associated with progression of arterial stiffness in the general population. ${ }^{112}$ Recent studies showed that evening chronotype may be associated with poor glycemic control in patient with T2DM. ${ }^{13}{ }^{14}$ Depressive status could also have a negative impact on glucose metabolism through increased counter-regulatory hormones. ${ }^{15}$ Eating late-night dinners may cause higher postprandial glucose levels in the following morning. ${ }^{16}$ In addition to those lifestyle habits, breakfast skipping is becoming a major potential target for preventing lifestyle-related diseases. Previous studies demonstrated relationships between breakfast skipping and increases in both body weight ${ }^{17}$ and risk of $\mathrm{T}_{2} \mathrm{DM}^{18}$ in the general population. A recent cross-sectional study demonstrated that breakfast skipping was associated with a higher prevalence of subclinical atherosclerosis in the general population. ${ }^{19}$ Furthermore, three cohort studies demonstrated that breakfast skipping was associated with increased risks of CVD and stroke in members of the general population without a history of CVD. ${ }^{20-22}$ Patients with T2DM are at high risk of developing CVD, ${ }^{1}$ but it has remained largely unknown whether like the general population, they are more likely to develop atherosclerosis if they skip breakfast.

As mentioned above, lifestyle habits are potential targets for primary CVD prevention strategies in addition to conventional atherosclerotic risk factors. However, there is limited literature addressing this issue in patients with T2DM. This is an exploratory study to bring insight on the lifestyle habits that are associated with longitudinal changes in arterial stiffness in patients with T2DM free of apparent CVD.

\section{METHOD}

Subjects

The subjects of this cohort study were recruited from the Diabetes Outpatient Clinic of Juntendo University (Tokyo, Japan), Secomedic Hospital (Funabashi, Japan) and Naka Kinen Clinic (Naka, Japan), as previously described. ${ }^{82324}$ This is an ongoing, observational, prospective cohort study that aims to investigate the relationships between lifestyle habits and the onset of CVD over an 8-year follow-up period. This study is an exploratory subanalysis of the cohort study. The study design, inclusion criteria and exclusion criteria have been published previously. ${ }^{82324}$ A total of 736 outpatients with T2DM and no history of apparent CVD were recruited between June 2013 and January 2014. However, two patients withdrew their consent.

\section{Questionnaire survey}

Questionnaire surveys were administered at baseline and at 2 and 5 years as previously described. ${ }^{8324}$ Sleep quality was evaluated with the use of the Pittsburgh Sleep Quality Index (PSQI). ${ }^{25}$ Based on PSQI scores, patients were divided into three groups as follows: the 'poor sleep quality group' had PSQI scores $\geq 9$, the 'average sleep quality group' had PSQI scores of 6-8 and the 'good sleep quality group' had PSQI scores $\leq 5 .{ }^{26}$ Morningness/eveningness was evaluated with the MorningnessEveningness Questionnaire (MEQ) ${ }^{27}$ Scores of $65-86$ were classified as morning type, scores of 53-64 as neither type and scores of $16-52$ as evening type. ${ }^{23}$ Depressionrelated symptoms were evaluated with the use of the Beck Depression Inventory (BDI)-II. ${ }^{28}$ Higher scores represent a depressive state. The dietary intake of $56 \mathrm{food}$ and beverage items was estimated with the use of brief, self-administered diet history questionnaire. ${ }^{29}$ Physical activity level was assessed with the use of the International Physical Activity Questionnaire (IPAQ). ${ }^{30}$

Subjects were asked about their average nightly sleep duration over the past month. Subjects were divided into three groups as follows: long sleep duration group, 8.5 hours or more; intermediate sleep duration group, $5.5-8.5$ hours and short sleep duration group, $<5.5$ hours. ${ }^{31}$ Subjects were also asked about excessive daytime sleepiness, as follows: "Have you ever had excessive daytime sleepiness?" Workers were defined as full-time employees or shift workers as previously described. ${ }^{82}{ }^{24}$ Subjects were asked whether they were current smokers or had smoked previously. They were classified as never-smokers, former smokers or current smokers.

Finally, the questionnaire addressed each individual's dietary schedule. Subjects were asked about the times at which they ate breakfast, lunch, dinner and snacks, as follows: "What time do you have each meal?" Subjects were asked about the number of meals they ate per day, as follows: "How many meals do you have per day? One, 2, 3, 4 or 5 or more". Subjects were asked about the number of regular snacks they ate per day, as follows: "How many times do snack per day? Almost never, 1, 2, 3,4 or 5 or more". Snacking after dinner was evaluated by the following question: "Do you eat fruits or other sweet snacks after dinner?" Subjects were asked about the amount of time between their last meal (either dinner or a snack) and bedtime. They were also asked about their average frequency of breakfast consumption, as follows: 
"How many times per week do you eat breakfast?" Finally, they were asked about the average frequency with which they ate dinner out, as follows: "How many times per week do you eat dinner out and/or eat ready-made dinners at home? Almost never, 1 to 2, 3 to 4 or 5 or more".

\section{Biochemical tests}

From baseline to 5 years, fasting blood samples were obtained from participants once per year. Hemoglobin A1c (HbA1c) (National Glycohemoglobin Standardization Program), liver and renal function tests, lipid levels and uric acid level were measured with standard techniques. Using a spot urine, sample urinary albumin excretion (UAE) was measured by latex agglutination assay. The eGFR was calculated by the formula. ${ }^{32}$

\section{Measurement of baPWV}

Using an automatic waveform analyzer (BP-203RPE; Colin Medical Technology, Komaki, Japan), baPWV was measured at baseline and at 2 and 5 years, as described previously. ${ }^{23}$ The average value of right baPWV and left baPWV was used as an individual representative value. The validity and reproducibility of baPWV measurements were confirmed in a previous study. ${ }^{33}$ The ankle-brachial index was also measured. The baPWV data of patients with a resting ankle-brachial index $\leq 0.90$ were excluded as they were considered to have peripheral artery disease.

\section{Statistical analysis}

Results are presented as mean $\pm \mathrm{SD}$ or median (IQR) for continuous variables, and as number (proportion) for categorical variables. Natural log-transformed values for triglycerides and UAE were used to approximate normal distribution. Patient characteristics, lifestyle habits and clinical variables over the study period were assessed with the mixed-effects model for repeated measures (MMRM), including time (years) as a factor with unstructured within-subject correlation. Longitudinal baPWV was analyzed using a linear mixed-effects model that included time, patient characteristics, lifestyle habits and possible atherosclerotic risk factors at baseline as fixed effects, and patient as a random effect to account for the inherent correlation of repeated measures on the same individual over time. We added the interaction term between time and each covariate in the mixed-effects model.

We further investigated the demographic and other characteristics of the subjects according to the frequency of breakfast intake at baseline. Since the majority of subjects ate breakfast 7 times per week, the rest of the subjects were almost equally divided into two groups for comparison based on the frequency of breakfast intake. Trend association across these three breakfast intake groups was evaluated by linear regression analysis for continuous variables and logistic regression analysis for categorical variables. The adjusted mean of baPWV was estimated using the MMRM method, taking into account the breakfast intake group, time, its interaction, baseline age and gender.

Statistical tests were two-sided with a $5 \%$ significant level. All analyses were performed using SAS software V.9.4 (SAS Institute, Cary, North Carolina, USA).

\section{RESULTS}

Patient demographic and background characteristics during the study period

At baseline, the data of 734 patients were available. Among them, a total of 99 subjects did not visit any of the study institutions at 5 years: 49 were transferred to another medical institution due to changes in work or house address, 45 subjects dropped out and 5 subjects died. The baseline clinical characteristics of the 734 patients with T2DM are summarized in table 1 . The mean age was $57.8 \pm 8.5$ years, $63 \%$ were male, $\mathrm{HbA1c}$ was $7.0 \% \pm 1.0 \%(52.6 \pm 10.9 \mathrm{mmol} / \mathrm{mol})$ and the estimated duration of T2DM was $9.9 \pm 7.2$ years. Over 5 years of follow-up, modest but significant increases were seen in HbA1c levels, and with respect to lifestyle habits, in both the MEQ score and PSQI score. With respect to conventional atherosclerotic risk factors, modest but significant changes occurred in BMI, systolic and diastolic BP, highdensity lipoprotein (HDL) cholesterol and logarithm of triglycerides. Renal function evaluated by eGFR and albuminuria slightly deteriorated, while baPWV significantly increased.

\section{Relationships between longitudinal brachial-ankle pulse wave velocity and lifestyle habits}

First, we used a linear mixed-effects model to examine the relationships between longitudinal baPWV, adjusted for age and gender and each lifestyle habit. We found a significant interaction between time and excessive daytime sleepiness (see online supplementary table S1), suggesting that baPWV in subjects with excessive daytime sleepiness significantly decreased with time compared with those without. On the other hand, there were no significant interactions between time and other lifestyle habits, including chronotype, sleep quality, sleep duration, depressive status, physical activity, caloric intake, alcohol consumption, smoking status, shift worker status, the frequency of breakfast skipping or time of dinner for longitudinal baPWV throughout the study period (see online supplementary tables S2-4; other data not shown). However, this analysis demonstrated that poor sleep quality was significantly associated with persistently high baPWV (see online supplementary table S2). Also, less frequent breakfast intake was significantly associated with persistently high baPWV (see online supplementary table S3). In addition, shift workers had significantly and persistently higher baPWV than non-shift workers (see online supplementary table S4).

\section{Predictors of longitudinal brachial-ankle pulse wave velocity} during 5 -year follow-up

Next, we aimed to identify lifestyle habits associated with longitudinal changes in baPWV using a multivariable 


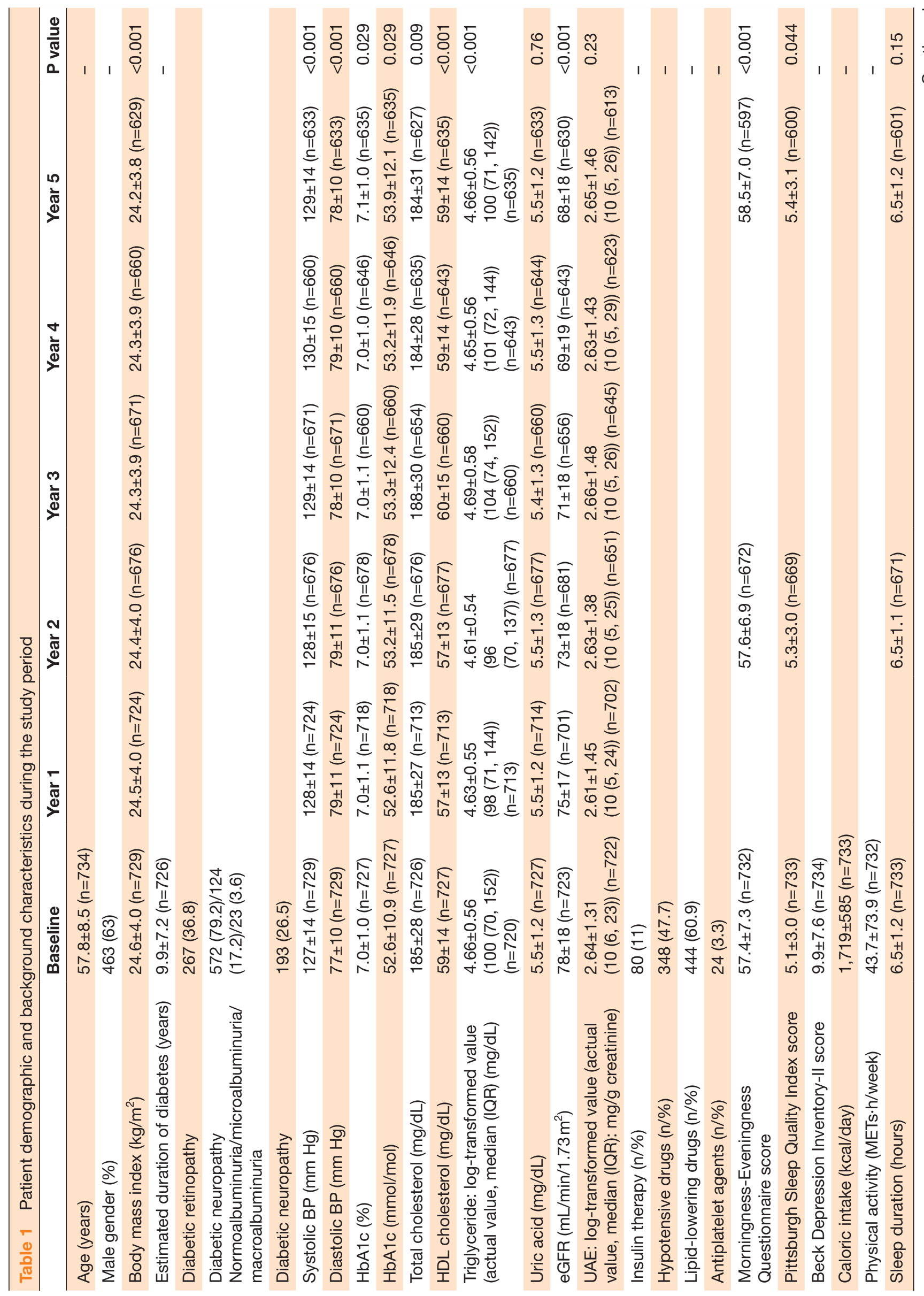


linear mixed-effects model adjusted for age and gender. As shown in table 2, the lower frequency of breakfast intake was significantly associated with persistently high baPWV, independent of other lifestyle habits. Although shift workers tended to have persistently higher baPWV than non-shift workers, and poor sleep quality tended to be associated with persistently higher baPWV than good sleep quality, lifestyle habits including excessive daytime sleepiness showed no significant association with longitudinal changes in baPWV. Furthermore, we investigated whether lifestyle habits in patients with T2DM were associated with persistently increased arterial stiffness independent of conventional atherosclerotic risk factors.

In a multivariable linear mixed-effects model that included both lifestyle habits and conventional atherosclerotic risk factors, the frequency of breakfast intake was still an independent predictive factor for persistently higher baPWV, in addition to older age, longer estimated duration of T2DM, lower BMI, higher systolic BP, higher logarithm of triglycerides, higher uric acid microalbuminuria and use of antiplatelet agents (table 3 ).

\section{Characteristics of each group at baseline by frequency of breakfast intake}

We further investigated subjects' demographic and other characteristics classified by the frequency of breakfast intake. As the majority of subjects ate breakfast 7 times a week, the remaining subjects were almost equally divided into two groups for comparison. According to this classification, the weekly breakfast intake was 7 times a week in 639 subjects, $<4$ times a week in 42 subjects and $\geq 4$ and $<7$ times a week in 52 subjects, as shown in table 4 .

Subjects who ate breakfast $<4$ times a week had significantly and persistently higher estimated baPWV than those who ate breakfast $\geq 4$ and $<7$ times a week and those who ate breakfast 7 times a week (figure 1). The baPWV in all three groups tended to increase throughout the study period. Even when replacing continuous variables with categorical variables in the multivariable linear mixedeffects model, subjects who ate breakfast $<4$ times a week had still persistently higher baPWV than those who ate breakfast $\geq 4$ and $<7$ times a week and those who ate breakfast daily (see online supplementary table 5). As shown in table 4 , subjects who ate breakfast $<4$ times a week tended to be young and obese; had a low mean MEQ score, high mean PSQI score and high mean BDI-II score; had high alcohol consumption and ate dinner late, frequently ate late-evening snacks and frequently ate out and/or ate ready-made meals at home for dinner. There were no significant trends in other lifestyle habits, including energy intake, sleep time and mean IPAQ scores. In terms of metabolic-renal parameters, patients who ate breakfast $<4$ times a week had higher HbAlc levels, higher diastolic BP, higher triglyceride levels, higher uric acid levels and less frequent nephropathy and neuropathy, and also tended to have lower HDL cholesterol levels (see online supplementary table 6). However, there were no significant differences in both frequency of nephropathy and 
Table 2 Predictors of longitudinal brachial-ankle pulse wave velocity during 5-year follow-up

\begin{tabular}{lllc}
\hline & Comparison & Regression coefficient (95\% Cl) & P value \\
\hline Intercept & & $838(423$ to 1254$)$ & $<0.001$ \\
\hline Time & 1 year & $23.0(19.9$ to 26.1$)$ & $<0.001$ \\
\hline Age & 1 year & $15.5(13.2$ to 17.8$)$ & $<0.001$ \\
\hline Gender & Male vs female & $39.3(-5.7$ to 84.2$)$ & 0.09 \\
\hline $\begin{array}{l}\text { Morningness-Eveningness } \\
\text { Questionnaire score }\end{array}$ & Neither type vs evening type & $30.1(-16.5$ to 76.8$)$ & 0.21 \\
\hline & Morning type vs evening type & $23.5(-40.1$ to 87.1$)$ & 0.47 \\
\hline Pittsburgh Sleep Quality Index score & Average vs good & $32.9(-12.1$ to 78.0$)$ & 0.15 \\
\hline & Poor vs good & $68.0(-1.33$ to 137.4$)$ & 0.05 \\
\hline Sleep duration & Intermediate vs short & $-11.6(-66.0$ to 42.8$)$ & 0.68 \\
\hline Beck Depression Inventory-II score & Long vs short & $14.1(-88.3$ to 117.1$)$ & 0.78 \\
\hline Caloric intake & 1 point & $-0.65(-3.20$ to 1.90$)$ & 0.62 \\
\hline Physical activity & 1 kcal/day & $-0.02(-0.06$ to 0.01$)$ & 0.18 \\
\hline Alcohol consumption & 1 METs-h/week & $-0.08(-0.32$ to 0.16$)$ & 0.52 \\
\hline Current smoker & 1 g/day & $0.49(-0.45$ to 1.44$)$ & 0.31 \\
\hline & Former smoker vs current smoker & $-27.4(-78.1$ to 22.5$)$ & 0.28 \\
\hline Frequency of breakfast intake & Former smoker vs & $-21.8(-71.5$ to 27.9$)$ & 0.39 \\
\hline Time of dinner & never-smoker & & \\
\hline Excessive daytime sleepiness & One time a week & $-25.3(-40.6$ to -10.0$)$ & 0.001 \\
\hline Shift worker & 1 hour & $-0.85(-18.5$ to 16.8$)$ & 0.92 \\
\hline & Yes vs no & $-21.1(-61.4$ to 19.3$)$ & 0.31 \\
\hline
\end{tabular}

Longitudinal brachial-ankle pulse wave velocity was analyzed with a linear mixed-effects model using time and lifestyle habits at baseline as fixed effects, and patient as a random effect.

neuropathy among three groups after adjusting for age, gender, duration of T2DM and HbA1c levels at baseline. Throughout the study period, subjects who ate breakfast $<4$ times a week tended to be obese with a low mean MEQ score and high mean PSQI score. Also, they tended to have higher triglyceride levels and uric acid levels, and lower HDL cholesterol levels, than subjects who ate breakfast $\geq 4$ and $<7$ times a week or 7 times a week.

\section{DISCUSSION}

In this study, we demonstrated for the first time that breakfast skipping was associated with persistently high baPWV in patients with T2DM. Although the mechanisms by which breakfast skipping induces arterial stiffness remain largely unknown, we propose the following possible scenarios. First, breakfast skipping causes a sensation of hunger, and leads to overeating later in the day, causing weight gain, insulin resistance and poor glycemic control. ${ }^{345}$ In this study, while the subjects who ate breakfast $<4$ times a week did not have a significantly different daily energy intake than subjects in the other two groups, they consumed more calories later in the day; specifically, they tended to eat late dinners, frequently ate late-evening snacks and often ate out and ate ready-made meals at home for dinner (table 4). A previous cross-sectional study reported that eating at night was positively associated with BMI in middle-aged subjects, ${ }^{36}$ and therefore greater daily energy intake late in the day may be associated with deterioration of metabolic controls through weight gain, leading to worsening of arterial stiffness. A second mechanism by which breakfast skipping may induce arterial stiffness is by causing overactivity in the hypothalamic-pituitary-adrenal axis due to prolonged fasting, resulting in elevation of $\mathrm{BP}^{37}$ Third, individuals who skip breakfast are more likely to make other unfavorable choices regarding lifestyle habits. In this study, subjects who ate breakfast $<4$ times a week tended to have an evening chronotype, poor sleep quality and more depression-related symptoms. Due to social norms, individuals with an evening chronotype may be forced to be awake at times they do not prefer, and this may cause stress that activates the sympathetic adrenal nervous system. ${ }^{38}$ Subsequent excess release of stress hormones, including cortisol and catecholamines, may lead to the progression of atherosclerosis. In addition, a previous report showed that high levels of catecholamines caused by poor sleep quality may be associated with progression of arterial stiffness in healthy middle-age adults. ${ }^{12}$ Thus, a cluster of unhealthy habits may contribute to persistently high baPWV in subjects who eat breakfast infrequently. 
Table 3 Results of multiple linear regression analysis for variables associated with longitudinal brachial-ankle pulse wave velocity during 5-year follow-up

\begin{tabular}{|c|c|c|c|}
\hline Variable & Comparison & Adjusted ORs $(95 \% \mathrm{Cl})$ & $P$ value \\
\hline Intercept & & $213(-281$ to 706$)$ & 0.40 \\
\hline Time (years) & 1 year & 23.3 (20.2 to 26.4$)$ & $<0.0001$ \\
\hline Age (years) & 1 year & 13.4 (10.9 to 15.9$)$ & $<0.0001$ \\
\hline Gender & Male vs female & $4.2(-41.2$ to 49.5$)$ & 0.86 \\
\hline \multirow[t]{2}{*}{ Morningness-Eveningness Questionnaire score } & Neither type vs evening type & $19.5(-23.3$ to 62.3$)$ & 0.37 \\
\hline & Morning type vs evening type & $19.2(-39.9$ to 78.4$)$ & 0.52 \\
\hline \multirow[t]{2}{*}{ Pittsburgh Sleep Quality Index score } & Average vs good & $32.5(-9.2$ to 74.3$)$ & 0.13 \\
\hline & Poor vs good & $42.7(-21.2$ to 106.5$)$ & 0.19 \\
\hline \multirow[t]{2}{*}{ Sleep duration } & Intermediate vs short & $-2.06(-51.8$ to 47.7$)$ & 0.93 \\
\hline & Long vs short & $-7.65(-104.1$ to 88.8$)$ & 0.88 \\
\hline Beck Depression Inventory-II score & 1 point & $-1.85(-4.25$ to 0.55$)$ & 0.13 \\
\hline Caloric intake & $1 \mathrm{kcal} /$ day & $-0.02(-0.05$ to 0.01$)$ & 0.22 \\
\hline Physical activity & $1 \mathrm{METs} \cdot \mathrm{h} /$ week & $-0.11(-0.34$ to 0.13$)$ & 0.36 \\
\hline Alcohol consumption & $1 \mathrm{~g} /$ day & $0.24(-0.70$ to 1.17$)$ & 0.62 \\
\hline \multirow[t]{2}{*}{ Current smoker } & $\begin{array}{l}\text { Former smoker vs current } \\
\text { smoker }\end{array}$ & $-8.19(-55.8$ to 39.4$)$ & 0.74 \\
\hline & $\begin{array}{l}\text { Former smoker vs } \\
\text { Never-smoker }\end{array}$ & $-5.97(-53.2$ to 41.2$)$ & 0.80 \\
\hline Frequency of breakfast intake & One time a week & $-25.5(-39.5$ to -11.5$)$ & 0.0004 \\
\hline Time of dinner & 1 hour & $-7.56(-24.1$ to 8.9$)$ & 0.37 \\
\hline Excessive daytime sleepiness & Yes vs no & $-13.8(-51.3$ to 23.7$)$ & 0.47 \\
\hline Shift worker & Yes vs no & 38.1 (-18.0 to 94.2$)$ & 0.18 \\
\hline Estimated duration of diabetes & 1 year & 3.59 (1.02 to 6.15$)$ & 0.006 \\
\hline Body mass index & $1 \mathrm{~kg} / \mathrm{m}^{2}$ & $-14.3(-19.4$ to -9.16$)$ & $<0.0001$ \\
\hline Systolic BP & $1 \mathrm{~mm} \mathrm{Hg}$ & 5.35 (4.09 to 6.62$)$ & $<0.0001$ \\
\hline $\mathrm{HbA1c}$ & $1 \mathrm{mmol} / \mathrm{mol}$ & $1.26(-0.51$ to 3.03$)$ & 0.16 \\
\hline Total cholesterol & $1 \mathrm{mg} / \mathrm{dL}$ & $0.23(-0.50$ to 0.96$)$ & 0.54 \\
\hline HDL cholesterol & $1 \mathrm{mg} / \mathrm{dL}$ & $-0.89(-2.51$ to 0.73$)$ & 0.28 \\
\hline Triglyceride: log-transformed value & 1 unit & 56.0 (13.9 to 98.0$)$ & 0.009 \\
\hline eGFR & $1 \mathrm{~mL} / \mathrm{min} / 1.73 \mathrm{~m}^{2}$ & $1.08(-0.04 .2 .19)$ & 0.06 \\
\hline Uric acid (mg/dL) & $1 \mathrm{mg} / \mathrm{dL}$ & $25.8(-9.27$ to 42.3$)$ & 0.002 \\
\hline Diabetic retinopathy & Yes vs no & 20.1 (-16.8 to 56.9$)$ & 0.29 \\
\hline \multirow[t]{2}{*}{ Diabetic nephropathy } & $\begin{array}{l}\text { Macroalbuminuria vs } \\
\text { normoalbuminuria }\end{array}$ & $25.5(-20.2$ to 71.3$)$ & 0.27 \\
\hline & $\begin{array}{l}\text { Microalbuminuria vs } \\
\text { normoalbuminuria }\end{array}$ & 100.6 (5.2 to 196.0$)$ & 0.04 \\
\hline Diabetic neuropathy & Yes vs no & -26.1 (-65.3 to 13.1) & 0.19 \\
\hline Insulin therapy & Yes vs no & $3.03(-56.5$ to 62.6$)$ & 0.92 \\
\hline Antihypertension drugs & Yes vs no & $-3.76(-39.5$ to 32.0$)$ & 0.84 \\
\hline Antihyperlipidemia drugs & Yes vs no & $-25.7(-61.0$ to 9.7$)$ & 0.16 \\
\hline Antiplatelet drugs & Yes vs no & 127.2 (33.8 to 220.7) & 0.008 \\
\hline Sleep apnea syndrome & Yes vs no & $1.68(-97.3$ to 100.6$)$ & 0.97 \\
\hline
\end{tabular}

Longitudinal brachial-ankle pulse wave velocity was analyzed with a linear mixed-effects model using time, lifestyle habits and possible atherosclerotic risk factors at baseline as fixed effects, and patient as a random effect.

BP, blood pressure; eGFR, estimated glomerular filtration rate; HbA1c, hemoglobin A1c; HDL, high-density lipoprotein. 
荡

๓

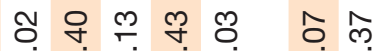

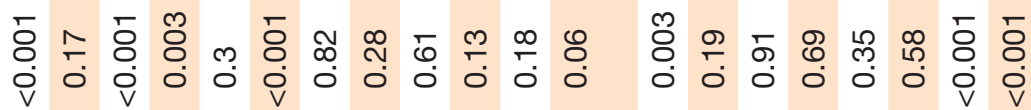

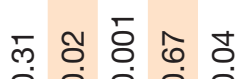

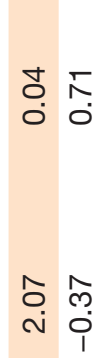

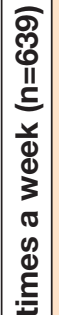
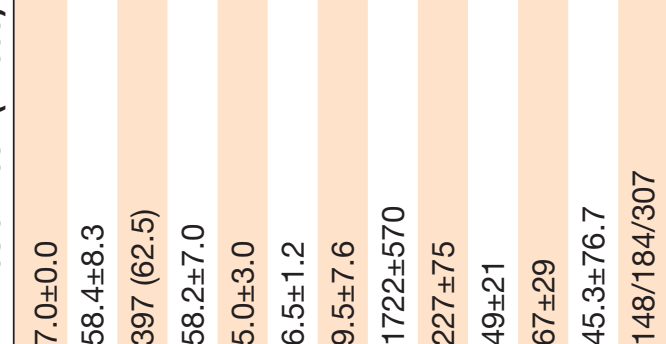

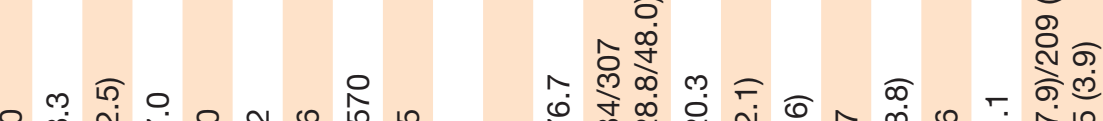

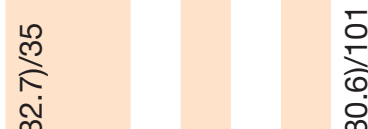

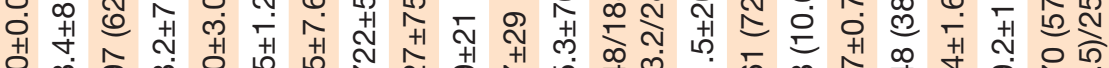
永

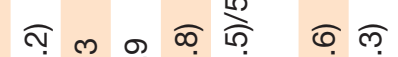

鱼贲年

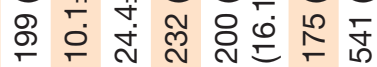

สิ์

$\stackrel{\oplus}{\frac{5}{0}}$

离

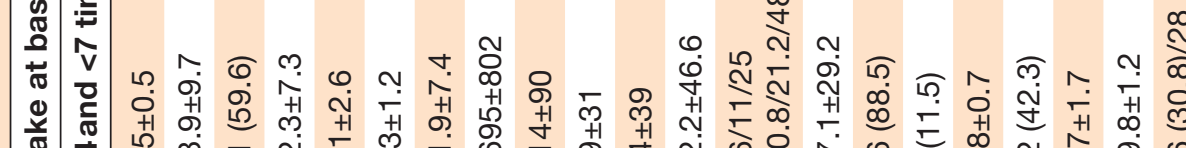

\section{$\stackrel{\widetilde{\pi}}{=}$ 苞 \\ 这

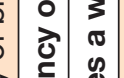

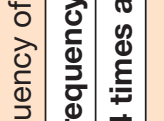

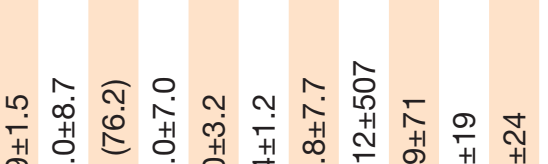

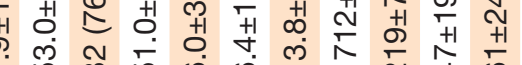

\section{i.}

$\stackrel{4}{2}$

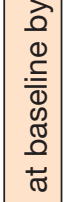

을

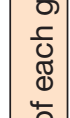

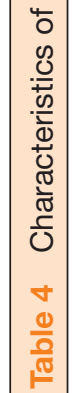

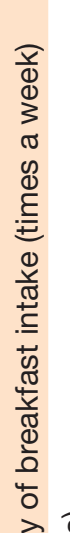

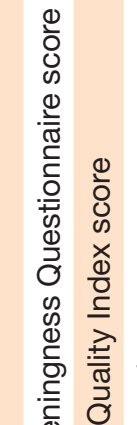

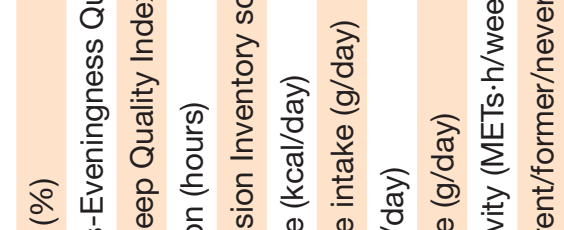

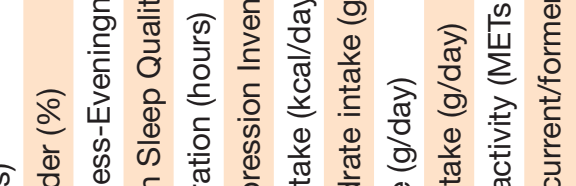

$\stackrel{\infty}{+\infty}$

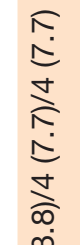

迎

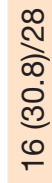

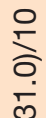

ma

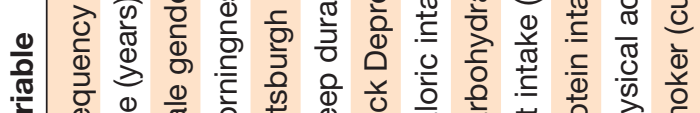




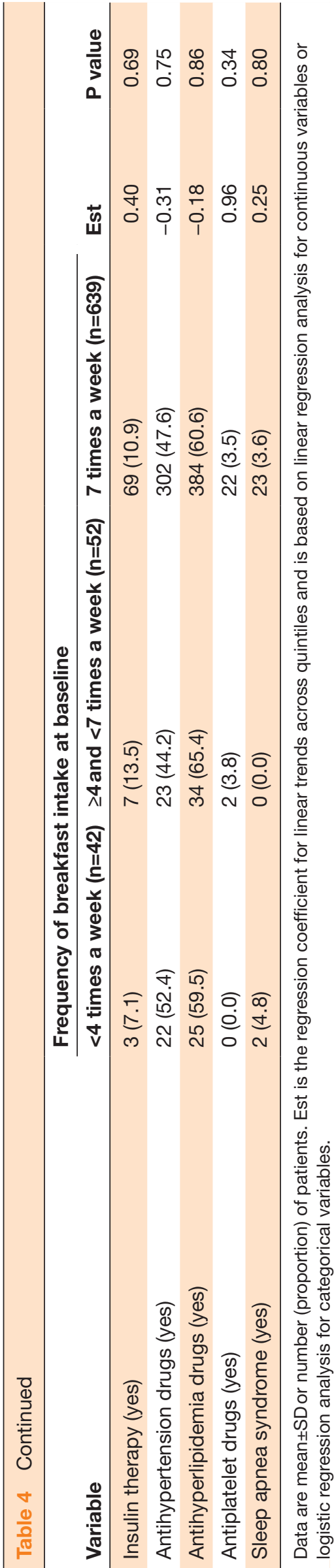

Fourth, subjects in this study who ate breakfast $<4$ times a week tended to be obese and had higher HbA1c levels, higher diastolic BP, higher triglyceride and uric acid levels and lower HDL cholesterol levels than subjects who ate breakfast more frequently. The accumulation of such conventional atherosclerotic risk factors could certainly contribute to the worsening of arterial stiffness.

More interestingly, even after adjusting for potential conventional atherosclerotic risk factors (table 3) and unfavorable lifestyle habits such as eating out and/or eating ready-made meal at home for dinner (data not shown), we found that a low frequency of breakfast intake was associated with persistently increased arterial stiffness in patients with T2DM. It is assumed that breakfast skipping affects additional atherosclerotic risk factors. For example, breakfast skipping impacts glucose regulation by reducing sensitization and activation of $\beta$-cells mediated by insulin and/or incretin. A previous study demonstrated that breakfast skipping affected postprandial glucose regulation throughout the day and was associated with impaired insulin secretion and lower incretin response in patients with $\mathrm{T}_{2} \mathrm{DM}{ }^{39}{ }^{39}$ In the same study, extended fasting as a result of breakfast skipping resulted in the prolonged elevation of plasma free fatty acids that caused insulin resistance. Therefore, conditions triggered by breakfast skipping may play an important role in the pathogenesis of arterial stiffness.

Since breakfast skipping was associated with increased arterial stiffness, one might expect that eating breakfast more frequently would improve arterial stiffness. Indeed, the above-mentioned study demonstrated that eating breakfast reversed the deleterious effects triggered by breakfast skipping. ${ }^{39}$ Unfortunately, our study design did not allow us to clarify how changes in breakfast-eating habits impact arterial stiffness.

\section{Limitations}

The present study has certain limitations. First, the observational cohort study design made it impossible to evaluate whether lifestyle habits and/or risk factors for atherosclerosis had a causal relationship with arterial stiffness. Second, we used only evaluation of arterial stiffness as a marker of CVD. In this regard, we are currently conducting a long-term follow-up study in the same cohort that focuses on lifestyle and onset of primary CVD. Third, we evaluated lifestyle using self-reported questionnaires. Although these have been widely used in many studies, the results could have been influenced by social desirability and recall bias. In particular, the frequency of breakfast intake was also self-reported, as in other studies, ${ }^{21}{ }^{22}$ and hence was prone to a subjective interpretation of the definition of 'breakfast'. Furthermore, the number of subjects who ate breakfast $<7$ times a week was relatively small. However, this proportion was not largely different from that of a recent study. ${ }^{40}$ Fourth, our data probably cannot be generalized to a broader population because we investigated a limited number of subjects and used specific inclusion and exclusion 
$(\mathrm{cm} / \mathrm{s})$

1800

$1770(1678,1861)$

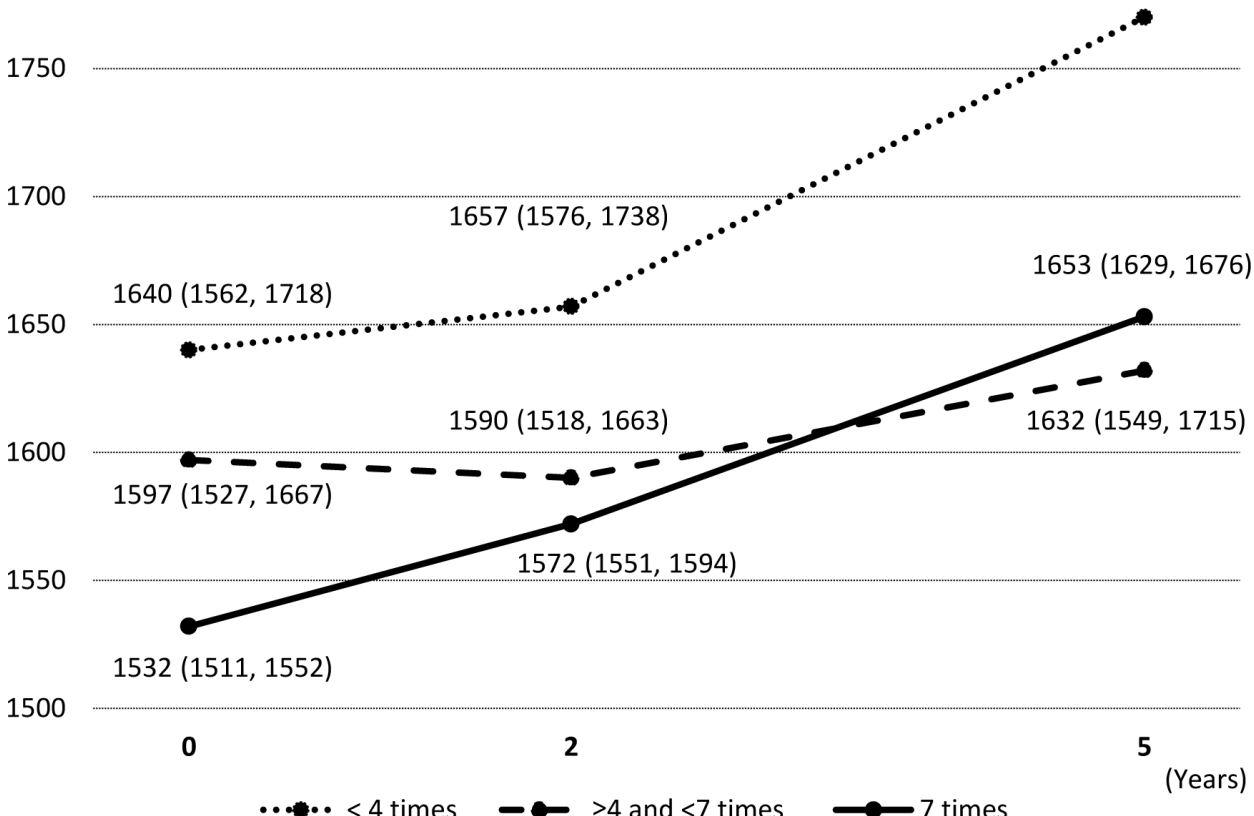

Figure 1 Adjusted means of brachial-ankle pulse wave velocity by breakfast intake group (95\% Cl: lower value and upper value) derived by a mixed-effects model for repeated measures method using the group, time, their interaction, baseline age and gender.

criteria.Fifth, there may be other lifestyle patterns that should be considered. Finally, the adherence of the drugs such as antidiabetes drugs, antihypertension drugs, antihyperlipidemia drugs and antiplatelet drugs that could be potential confounding factors should be considered. Especially, drug adherence may decrease in the individuals who skip breakfast because they are more likely to make other unfavorable choices.

\section{CONCLUSIONS}

In conclusion, the present study demonstrated that breakfast skipping was a lifestyle habit that was independently associated with persistently increased arterial stiffness. Therefore, asking about the frequency of breakfast intake in clinical practice may be a simple and useful method to help identify patients at high risk of developing CVD.

\section{Author affiliations}

${ }^{1}$ Department of Metabolism \& Endocrinology, Juntendo University Graduate School of Medicine, Bunkyo-ku, Japan

${ }^{2}$ Center for Molecular Diabetology, Juntendo University Graduate School of Medicine, Tokyo, Japan

${ }^{3}$ Naka Kinen Clinic, Naka, Japan

${ }^{4}$ Department of Biostatistics, Faculty of Medicine, University of Tsukuba, Tsukuba, Japan

${ }^{5}$ Center for Therapeutic Innovations in Diabetes, Juntendo University Graduate School of Medicine, Tokyo, Japan

${ }^{6}$ Sportology Center, Juntendo University Graduate School of Medicine, Tokyo, Japan

Acknowledgements The authors would like to thank the following staff who participated in this trial and provided excellent technical support: Yayoi Uchiyama and Emi Ito (Department of Metabolism and Endocrinology, Juntendo University Graduate School of Medicine), and Satako Douguchi, Norie Watahiki, Nana
Shiozawa, Misato Ojima, Akiko Haginoya and Katuhiro Kawauchi (Naka Kinen Clinic).

Contributors All authors contributed to the study design and were involved in all stages of manuscript development. TM and YO drafted the manuscript. MG, a statistician, was primarily responsible for data analysis. TM, YO, TO, MS, SN, YS, HI, MG and HW also collected, analyzed and interpreted the data, reviewed and edited the manuscript and approved the final manuscript. HW is the principal guarantor of this work; he has full access to all study data and takes responsibility for the integrity of the data and the accuracy of data analysis. All authors have read and agreed to the publication of the manuscript.

Funding This study was conducted through a research grant from the Manpei Suzuki Diabetes Foundation (to TM).

Disclaimer This funding agency had no role in study design, data collection or analysis, decision to publish or preparation of the manuscript.

Competing interests None declared.

Patient consent for publication Not required.

Ethics approval The study was approved by the institutional review board of Juntendo University Hospital and conducted in accordance with the principles described in the Declaration of Helsinki. All patients provided written informed consent prior to participation.

Provenance and peer review Not commissioned; externally peer reviewed.

Data availability statement Data are available on reasonable request. The analyzed datasets are available from the corresponding author (tom-m@juntendo. ac.jp) on reasonable request.

Open access This is an open access article distributed in accordance with the Creative Commons Attribution Non Commercial (CC BY-NC 4.0) license, which permits others to distribute, remix, adapt, build upon this work non-commercially, and license their derivative works on different terms, provided the original work is properly cited, appropriate credit is given, any changes made indicated, and the use is non-commercial. See: http://creativecommons.org/licenses/by-nc/4.0/.

ORCID iD

Tomoya Mita http://orcid.org/0000-0002-4401-2965 


\section{REFERENCES}

1 Haffner SM, Lehto S, Rönnemaa T, et al. Mortality from coronary heart disease in subjects with type 2 diabetes and in nondiabetic subjects with and without prior myocardial infarction. N Engl J Med 1998;339:229-34.

2 Taniwaki H, Kawagishi T, Emoto M, et al. Correlation between the intima-media thickness of the carotid artery and aortic pulse-wave velocity in patients with type 2 diabetes. vessel wall properties in type 2 diabetes. Diabetes Care 1999;22:1851-7.

3 Stehouwer CDA, Henry RMA, Ferreira I. Arterial stiffness in diabetes and the metabolic syndrome: a pathway to cardiovascular disease. Diabetologia 2008;51:527-39.

4 Nakamura M, Yamashita T, Yajima J, et al. Brachial-ankle pulse wave velocity as a risk stratification index for the short-term prognosis of type 2 diabetic patients with coronary artery disease. Hypertens Res 2010;33:1018-24.

5 Maeda Y, Inoguchi T, Etoh E, et al. Brachial-ankle pulse wave velocity predicts all-cause mortality and cardiovascular events in patients with diabetes: the Kyushu prevention study of atherosclerosis. Diabetes Care 2014;37:2383-90.

6 Ferreira MT, Leite NC, Cardoso CRL, et al. Correlates of aortic stiffness progression in patients with type 2 diabetes: importance of glycemic control: the Rio de Janeiro type 2 diabetes cohort study. Diabetes Care 2015;38:897-904.

7 Fukui M, Tanaka M, Shiraishi E, et al. Serum uric acid is associated with microalbuminuria and subclinical atherosclerosis in men with type 2 diabetes mellitus. Metabolism 2008;57:625-9.

8 Osonoi Y, Mita T, Osonoi T, et al. Poor sleep quality is associated with increased arterial stiffness in Japanese patients with type 2 diabetes mellitus. BMC Endocr Disord 2015;15:29.

9 Hamamura M, Mita T, Osonoi Y, et al. Relationships among conventional cardiovascular risk factors and lifestyle habits with arterial stiffness in type 2 diabetic patients. J Clin Med Res 2017;9:297-302

10 Funck KL, Laugesen E, Høyem P, et al. Low physical activity is associated with increased arterial stiffness in patients recently diagnosed with type 2 diabetes. Am J Hypertens 2016;29:882-8.

11 Haack M, Sanchez E, Mullington JM. Elevated inflammatory markers in response to prolonged sleep restriction are associated with increased pain experience in healthy volunteers. Sleep 2007;30:1145-52.

12 Zhang J, Ma RCW, Kong APS, et al. Relationship of sleep quantity and quality with 24-hour urinary catecholamines and salivary awakening cortisol in healthy middle-aged adults. Sleep 2011;34:225-33.

13 Iwasaki M, Hirose T, Mita T, et al. Morningness-eveningness questionnaire score correlates with glycated hemoglobin in middleaged male workers with type 2 diabetes mellitus. J Diabetes Investig 2013;4:376-81.

14 Reutrakul S, Hood MM, Crowley SJ, et al. Chronotype is independently associated with glycemic control in type 2 diabetes. Diabetes Care 2013;36:2523-9.

15 Musselman DL, Betan E, Larsen $\mathrm{H}$, et al. Relationship of depression to diabetes types 1 and 2: epidemiology, biology, and treatment. Biol Psychiatry 2003;54:317-29.

16 Tsuchida Y, Hata S, Sone Y. Effects of a late supper on digestion and the absorption of dietary carbohydrates in the following morning. $J$ Physiol Anthropol 2013;32:9.

17 van der Heijden AAWA, Hu FB, Rimm EB, et al. A prospective study of breakfast consumption and weight gain among U.S. men. Obesity 2007;15:2463-9.

18 Mekary RA, Giovannucci E, Willett WC, et al. Eating patterns and type 2 diabetes risk in men: breakfast omission, eating frequency, and snacking. Am J Clin Nutr 2012;95:1182-9.
19 Uzhova I, Fuster V, Fernández-Ortiz A, et al. The importance of breakfast in atherosclerosis disease: insights from the PESA study. $J$ Am Coll Cardiol 2017;70:1833-42.

20 Cahill LE, Chiuve SE, Mekary RA, et al. Prospective study of breakfast eating and incident coronary heart disease in a cohort of male US health professionals. Circulation 2013;128:337-43.

21 Kubota Y, Iso H, Sawada N, et al. Association of breakfast intake with incident stroke and coronary heart disease: the Japan public health Center-Based study. Stroke 2016;47:477-81.

22 Rong S, Snetselaar LG, Xu G, et al. Association of skipping breakfast with cardiovascular and all-cause mortality. J Am Coll Cardiol 2019;73:2025-32.

23 Osonoi Y, Mita T, Osonoi T, et al. Morningness-eveningness questionnaire score and metabolic parameters in patients with type 2 diabetes mellitus. Chronobiol Int 2014;31:1017-23.

24 Osonoi Y, Mita T, Osonoi T, et al. Relationship between dietary patterns and risk factors for cardiovascular disease in patients with type 2 diabetes mellitus: a cross-sectional study. Nutr J 2016;15:15.

25 Buysse DJ, Reynolds CF, Monk TH, et al. The Pittsburgh sleep quality index: a new instrument for psychiatric practice and research. Psychiatry Res 1989;28:193-213.

26 Tsai Y-W, Kann N-H, Tung T-H, et al. Impact of subjective sleep quality on glycemic control in type 2 diabetes mellitus. Fam Pract 2012;29:30-5.

27 Horne JA, Ostberg O. A self-assessment questionnaire to determine morningness-eveningness in human circadian rhythms. Int $J$ Chronobiol 1976;4:97-110.

28 Beck AT, Steer RA, Brown GK. Manual for the Beck depression Inventory-II. San Antonio, TX: Psychological Corporation, 1996.

29 SaT A. Standard tables of food composition in Japan (in Japanese). 5th rev edn. Tokyo: Printing Bureau of the Ministry of Finance, 2005.

30 Craig CL, Marshall AL, Sjöström M, et al. International physical activity questionnaire: 12 -country reliability and validity. Med Sci Sports Exerc 2003;35:1381-95.

31 Ohkuma T, Fujii $\mathrm{H}$, Iwase $\mathrm{M}$, et al. Impact of sleep duration on obesity and the glycemic level in patients with type 2 diabetes: the Fukuoka diabetes registry. Diabetes Care 2013;36:611-7.

32 Matsuo S, Imai E, Horio M, et al. Revised equations for estimated GFR from serum creatinine in Japan. Am J Kidney Dis 2009;53:982-92.

33 Yamashina A, Tomiyama $\mathrm{H}$, Takeda K, et al. Validity, reproducibility, and clinical significance of noninvasive brachial-ankle pulse wave velocity measurement. Hypertens Res 2002;25:359-64.

34 Astbury NM, Taylor MA, Macdonald IA. Breakfast consumption affects appetite, energy intake, and the metabolic and endocrine responses to foods consumed later in the day in male habitual breakfast eaters. J Nutr 2011;141:1381-9.

35 Reutrakul S, Hood MM, Crowley SJ, et al. The relationship between breakfast skipping, chronotype, and glycemic control in type 2 diabetes. Chronobiol Int 2014;31:64-71.

36 Meule A, Allison KC, Brähler E, et al. The association between night eating and body mass depends on age. Eat Behav 2014;15:683-5.

37 Witbracht M, Keim NL, Forester S, et al. Female breakfast skippers display a disrupted cortisol rhythm and elevated blood pressure. Physiol Behav 2015;140:215-21.

38 Lucassen EA, Zhao X, Rother KI, et al. Evening chronotype is associated with changes in eating behavior, more sleep apnea, and increased stress hormones in short sleeping obese individuals. PLOS One 2013;8:e56519.

39 Jakubowicz D, Wainstein J, Ahren B, et al. Fasting until noon triggers increased postprandial hyperglycemia and impaired insulin response after lunch and dinner in individuals with type 2 diabetes: a randomized clinical trial. Diabetes Care 2015;38:1820-6.

40 Sakai R, Hashimoto Y, Ushigome E, et al. Late-night-dinner is associated with poor glycemic control in people with type 2 diabetes: the KAMOGAWA-DM cohort study. Endocr $J$ 2018;65:395-402. 